\title{
Calcium Pyrophosphate Dihydrate Deposition Disease in Young Patients: Two Case Reports
}

\author{
Geetha WICKREMATILAKE \\ Department of Rheumatology, District General Hospital, Nuwara Eliya, Nuwara Eliya, Sri Lanka
}

\begin{abstract}
Calcium pyrophosphate deposition disease (CPDD) is a type of arthritis caused by the deposition of calcium pyrophosphate crystals, and may present as either acute or chronic arthritis. Development of CPPD crystal deposition disease in young people may be associated with metabolic diseases such as hemochromatosis, hyperparathyroidism, hypomagnesemia, Wilson's disease, hypothyroidism, gout, acromegaly, and X-linked hypophosphatemic rickets. Therefore, investigations for a predisposing metabolic condition are advised in young-onset polyarticular CPPD crystal deposition disease. In this article, we report two young patients who were investigated for recurrent joint pain due to CPPD disease.

Keywords: Calcium pyrophosphate dihydrate; chondrocalcinosis; pseudogout.
\end{abstract}

Calcium pyrophosphate dihydrate (CPPD) crystal deposition disease is characterized by the deposition of CPPD crystals in the articular cartilage visualized as intra-articular calcifications [chondrocalcinosis (CC)], with identification of the crystals in the synovial fluid, and an acute arthropathy called pseudogout. ${ }^{1}$

Patients with CPPD disease may be asymptomatic or present with a range of symptoms and signs similar to gout or other forms of inflammatory arthritis. However, attacks of pseudogout tend to be less painful and take longer to reach peak intensity than gout. ${ }^{2}$

Chondrocalcinosis occurs in three forms: a primary hereditary form, a form associated with metabolic disorders, and a sporadic form, which may in some cases represent the hereditary form. ${ }^{3,4}$

Molecular genetics studies have identified two genetic locations for familial CC: one related to a mutation in the long arm of chromosome 8 (8q), and the other resulting from a mutation on the short arm of chromosome 5 (5p). ${ }^{5}$ An autosomal dominant mode of inheritance was suggested in several reports. ${ }^{2,6} \mathrm{CC}$ is age related with an incidence of $5 \%$ in patients $>70$ years, and nearly $50 \%$ in patients $>90$ years. ${ }^{7}$ A primary metabolic disorder or familial predisposition should be considered if $\mathrm{CC}$ occurs in patients $<55$ years, or if there is florid polyarticular CC. $1,8,9$

An acute episode of CPPD disease may occur spontaneously or develop after trauma, surgery or a severe medical illness, similar to an attack of gout. Knee is the most commonly involved joint, followed by the wrist, metacarpophalangeal, hips, shoulder, and ankle joints. ${ }^{10}$ It is polyarticular in approximately two-thirds of patients, and often occurs in an asymmetric pattern. ${ }^{11}$ Rarely, there may be deposition of CPPD crystals in other parts of the skeleton, e.g. in the spine, symphysis pubis or temporomandibular joint. ${ }^{9}$ CPPD can also be associated with tendinitis, tenosynovitis and bursitis. $^{8,12}$ 
The gold standard for a definitive diagnosis of CPPD disease is the identification of CPPD crystals in synovial fluid. The characteristic crystals found in CPPD disease are generally rectangular, square, rhomboid or rod-shaped and can be seen using polarized light microscopy. ${ }^{11,13}$ Linear or punctate calcification of the hyaline and fibrocartilage is the characteristic finding on plain X-ray in $\mathrm{CC}^{14}$

\section{CASE REPORT}

Case 1- A 35-year-old female patient was admitted to the hospital with an episode of community acquired pneumonia. During her stay at the hospital, she developed a right knee joint pain and an effusion. Joint sepsis was excluded by an orthopedic surgeon. However, during her stay, she complained of pain in both knees and wrist joints needing a rheumatology consultation.

She gave a history of recurrent pain and swelling of her right knee joint and wrists for the past five years and was treated symptomatically by a general practitioner. These episodes were selflimiting and resolved usually within two to three weeks. There was a family history of her father and younger sister having similar problems and her father (64 years old) was being followed-up at

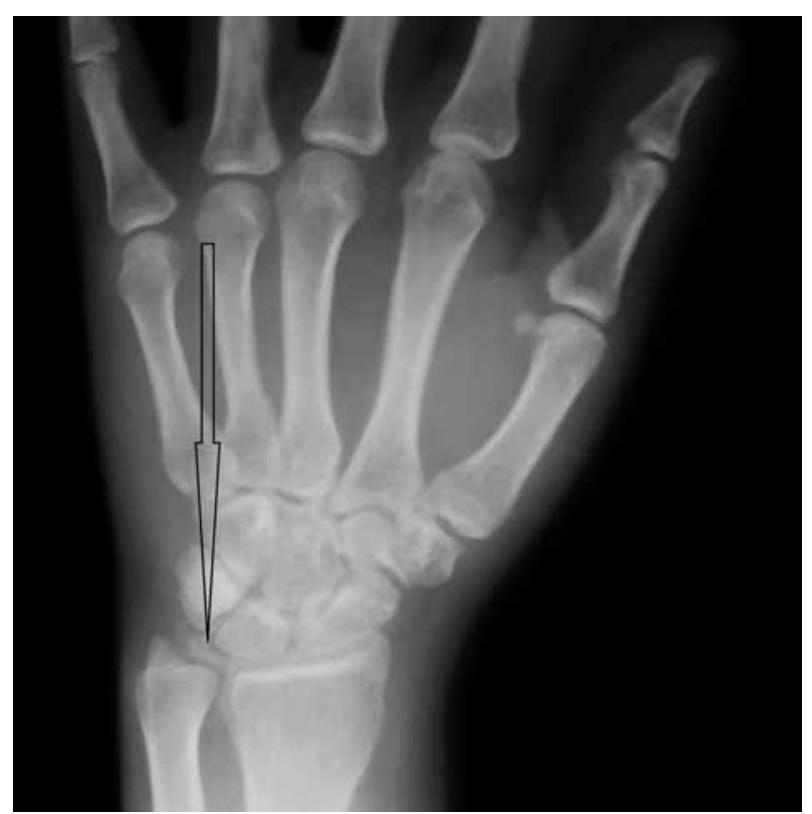

Figure 1. Wrists showed chondrocalcinosis of triangular fibrocartilage (Case 1). the clinic for CC. A written informed consent was obtained from the patient.

On examination, there was swelling and painful restriction of movement in her right knee. Imaging revealed calcification of cartilage of wrists, knee joints and hip joints although no microscopic confirmation was possible at this stage (Figure 1-4). She was investigated for a possible cause for $\mathrm{CC}$, although investigations to screen for a metabolic cause turned out to be negative with a possibility for hereditary CC.

Case 2- A 39-year-old male patient presented with swelling of both knee joints. He also gave a history of recurrent painful swelling of knee joints and wrist joints for more than 10 years and was treated by an Ayurvedic physician. Other medical history or family history of medical problems were insignificant. There was a massive swelling of both knee joints with effusions, which was aspirated and the X-rays revealed calcification of the articular cartilages (Figure 5). A written informed consent was obtained from the patient.

His hand X-rays showed hook like projections arising from radial aspect of second and third metacarpal heads with scapholunate advanced collapse and indentation of distal radius by the scaphoid bone. It also showed $\mathrm{CC}$ of the triangular fibrocartilage (Figure 6).

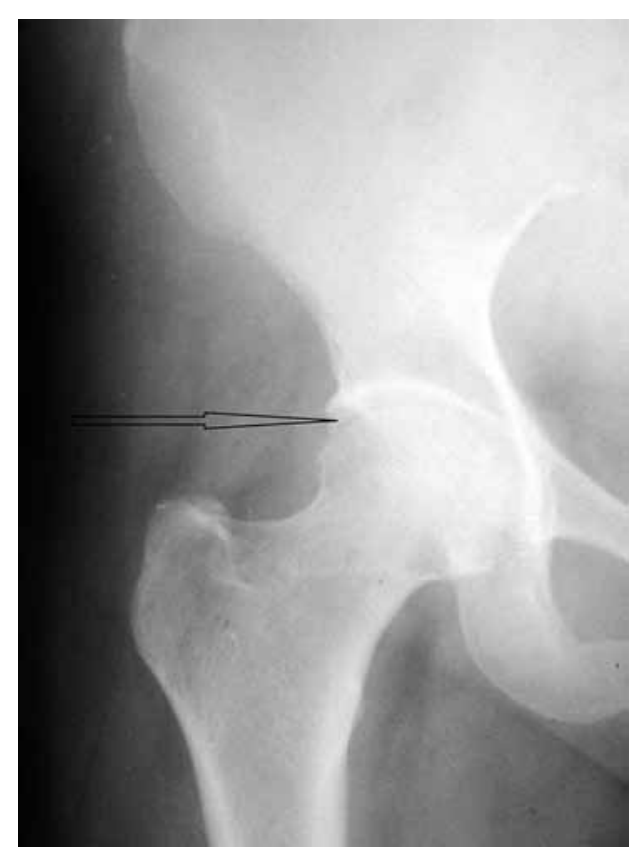

Figure 2. Chondrocalcinosis of hip (Case 1). 


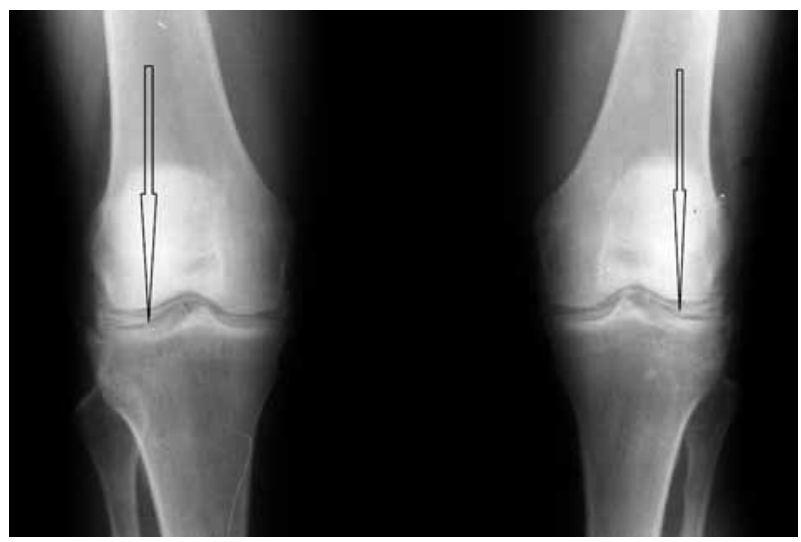

Figure 3. Knee joints with calcification of menisci (Case 1).

He had chondrocalcinosis involving the menisci and hyaline cartilage, with predominant patellofemoral joint osteoarthritis. His blood investigations to find a metabolic cause for $\mathrm{CC}$ were negative.

\section{DISCUSSION}

To the best of our knowledge, only a few cases of pseudogout have been reported in the literature in young patients. Development of CPPD crystal deposition disease in young people $(<55$ years) may be associated with metabolic diseases such as hemochromatosis,

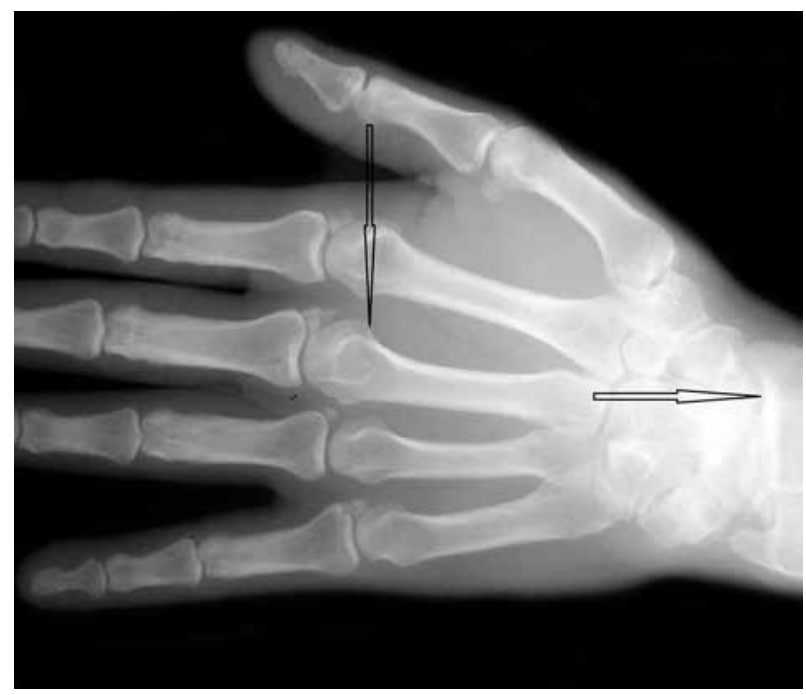

Figure 5. Hands show beaklike projections from second and third metacarpal heads and scapholunate advanced collapse (Case 2).

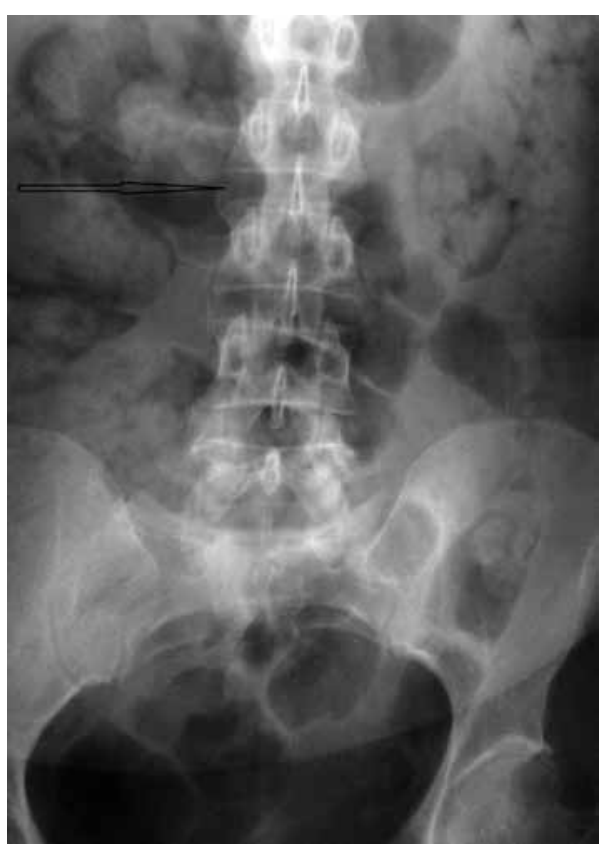

Figure 4. Chondrocalcinosis of annulus (Case 1).

hyperparathyroidism, hypomagnesemia, Wilson's disease, hypothyroidism, gout, acromegaly, and $\mathrm{X}$-linked hypophosphatemic rickets. Unlike in gout, there is currently no effective treatment that reduces or removes calcium pyrophosphate crystals from a joint. ${ }^{11,15}$

Aspiration of an affected joint not only provides fluid for confirmation of the diagnosis but also exclusion of infection and temporary relief from pain due to a reduction in the fluid volume within the joint. An intra-articular injection of corticosteroid can be very effective in reducing symptoms in patients who have

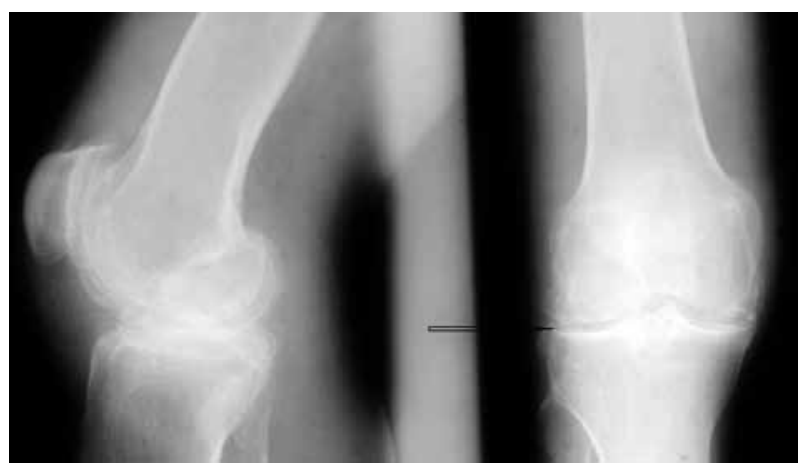

Figure 6. Knees show chondrocalcinosis involving the menisci and predominant patellofemoral joint osteoarthritis (Case 2). 
a single affected joint. Furthermore, an oral nonsteroidal anti-inflammatory drug or low dose colchicine may be effective in reducing acute symptoms. ${ }^{15} \mathrm{~A}$ short tapering course of oral corticosteroids may also provide relief from acute symptoms, especially in severe polyarticular attacks. ${ }^{9,15}$

Low dose colchicine $(0.5 \mathrm{mg}$, once or twice daily) may be effective in reducing the number of attacks with frequent recurrent episodes; however, there is no evidence on the use of nonsteroidal anti-inflammatory drugs in chronic CPPD crystal inflammatory arthritis. ${ }^{15}$

Methotrexate has been attempted only in patients with pseudorheumatoid presentation. ${ }^{16}$ Anakinra may have a role in controlling flairs of CPPD when conventional therapies are contraindicated or ineffective. ${ }^{17}$

Our patients were managed with steroid injections and nonsteroidal anti-inflammatory drug for acute attacks and physiotherapy to reduce pain and keep the joints flexible.

In conclusion, we reported two rare cases of CPPD crystal deposition disease in young patients. On screening, patients did not have any metabolic cause for pseudogout, although genetic predisposing could not be ruled out in the first patient. Second patient was a clear sporadic case of $\mathrm{CC}$ which is rare in the literature.

\section{Declaration of conflicting interests}

The author declared no conflicts of interest with respect to the authorship and/or publication of this article.

\section{Funding}

The author received no financial support for the research and/or authorship of this article.

\section{REFERENCES}

1. McCarty DJ. Calcium pyrophosphate dihydrate crystal deposition disease--1975. Arthritis Rheum 1976;19:275-85.

2. Ahn JK, Kim HJ, Kim EH, Jeon $\mathrm{CH}$, Cha HS, $\mathrm{Ha} \mathrm{CW}$, et al. Idiopathic calcium pyrophosphate dihydrate (CPPD) crystal deposition disease in a young male patient: a case report. J Korean Med Sci 2003;18:917-20.

3. Hughes AE, McGibbon D, Woodward E, Dixey J, Doherty M. Localisation of a gene for chondrocalcinosis to chromosome 5p. Hum Mol Genet 1995;4:1225-8.

4. Richette P, Bardin T, Doherty M. An update on the epidemiology of calcium pyrophosphate dihydrate crystal deposition disease. Rheumatology (Oxford) 2009;48:711-5.

5. Netter P, Bardin T, Bianchi A, Richette P, Loeuille D. The ANKH gene and familial calcium pyrophosphate dihydrate deposition disease. Joint Bone Spine 2004:71:365-8.

6. vander KorstJK, Geerards J, DriessensFC. A hereditary type of idiopathic articular chondrocalcinosis. Survey of a pedigree. Am J Med 1974;56:307-14.

7. Al-Tubaikh JA. CPPD and HADD. Internal Medicine. An Illustrated Radiological Guide. Chapter 47. Berlin Heidelberg; Springer-Verlag; 2010. p. 265-8.

8. Zhang W, Doherty M, Bardin T, Barskova V, Guerne PA, Jansen TL, et al. European League Against Rheumatism recommendations for calcium pyrophosphate deposition. Part I: terminology and diagnosis. Ann Rheum Dis 2011;70:563-70.

9. Abhishek A, Doherty M. Pathophysiology of articular chondrocalcinosis--role of ANKH. Nat Rev Rheumatol 2011;7:96-104.

10. Doherty M, Dieppe P. Clinical aspects of calcium pyrophosphate dihydrate crystal deposition. Rheum Dis Clin North Am 1988;14:395-414.

11. Longo D, Fauci A, Kasper D. Gout and other crystalassociated arthropathies. Harrison's Principles of Internal Medicine. Chapter 333. 18th ed. New York: McGraw Hill Medical; 2012. p. 2839-40.

12. Perera C, Brook A, Tynns K. Gout and other crystal arthropathies - Part 2. Australian Doctor 2009;21-9.

13. Macmullan P, McCarthy G. Treatment and management of pseudogout: insights for the clinician. Ther Adv Musculoskelet Dis 2012;4:121-31.

14. Dalbeth N, McQueen FM. Use of imaging to evaluate gout and other crystal deposition disorders. Curr Opin Rheumatol 2009;21:124-31.

15. Zhang W, Doherty M, Pascual E, Barskova V, Guerne PA, Jansen TL, et al. EULAR recommendations for calcium pyrophosphate deposition. Part II: management. Ann Rheum Dis 2011;70:571-5.

16. Ea HK, Lioté F. Calcium pyrophosphate dihydrate and basic calcium phosphate crystal-induced arthropathies: update on pathogenesis, clinical features, and therapy. Curr Rheumatol Rep 2004;6:221-7.

17. Ottaviani S, Brunier L, Sibilia J, Maurier F, Ardizzone $\mathrm{M}$, Wendling $\mathrm{D}$, et al. Efficacy of anakinra in calcium pyrophosphate crystal-induced arthritis: a report of 16 cases and review of the literature. Joint Bone Spine 2013;80:178-82. 